\title{
La fin de la crise ? Pour un dépassement phénoménologique du modèle critique
}

Par ANNABELLE DUFOURCQ

Université Charles de Prague

\section{Introduction}

Lorsqu'en 1935-1936 Husserl diagnostique une crise de la culture européenne et, au-delà, de l'humanité, une sorte d'angoisse semble envahir la phénoménologie ( La phénoménologie comme science, comme science sérieuse, rigoureuse, et même apodictiquement rigoureuse: ce rêve est fini $\left.{ }^{1}\right)$, couplée cependant avec des intonations prophétiques d'une positivité écrasante : "Combattons en tant que "bons Européens" contre ce danger des dangers [le naturalisme $][\ldots]$ et nous verrons alors sortir [...] des cendres de la grande lassitude, le phénix ressuscité d'une nouvelle vie intérieure et d'un nouveau souffle spirituel, gage d'un grand et long avenir pour l'humanité : car l'esprit seul est immortel $»^{2}$. Comme si Husserl ne parvenait à introduire

1 «Philosophie als Wissenschaft, als ernstliche, strenge, ja apodiktisch strenge Wissenschaft — der Traum ist ausgetraümt » (Edmund Husserl, Husserliana VI. Die Krisis der europäischen Wissenschaften und die transzendentale Phänomenologie. Eine Einleitung in die phänomenologische Philosophie, W. Biemel (Hrsg.), Den Haag, Martinus Nijhoff, 1954, p. 508 ; trad. fr. G. Granel, La crise des sciences européennes et la phénoménologie transcendantale, Paris, Éditions Gallimard, 1976, p. 562).

2 «Kämpfen wir gegen diese Gefahr der Gefahren als “gute Europäer” in jener Tapferkeit [...], dann wird aus [...] der Asche der großen Müdigkeit der Phoenix einer neuen Lebensinnerlichkeitdem und Vergeistigung auferstehen, als Unterpfand einer großen und fernen Menschenzukunft: Denn der Geist allein ist unsterblich » 
le séisme de la crise au cœur de sa philosophie qu'en lui donnant une forme policée reconduisant en fait à l'esprit rationaliste qui constitue toujours un des motifs majeurs de son projet. Nous souhaitons montrer que le séisme est pourtant bien là, avec une authentique radicalité, mais que ce n'est pas au concept de crise qu'il faut demander d'en rendre compte.

On trouve dans les développements husserliens de 1935-1936 tous les traits essentiels du concept général et classique de crise : ainsi que le montre l'image du phénix, au-delà du constat d'un sentiment d'absurdité, de perte de confiance dans des référents absolus, le modèle conceptuel de la crise implique l'idée qu'un tel malaise est un moment de tension paroxystique et qu'il annonce la nécessité d'une issue prochaine, laquelle est essentiellement

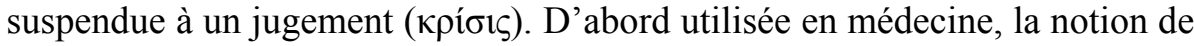
crise sous-entend classiquement que le désordre, le vacillement du sens et des valeurs sont une maladie, qu'ils sont forcément ponctuels et ouvrent sur deux possibilités imminentes : la déchéance irrémédiable ou la santé recouvrée. Cette notion suggère également que de tels déséquilibres malheureux font signe vers une solution fondée sur une analyse clairvoyante des origines

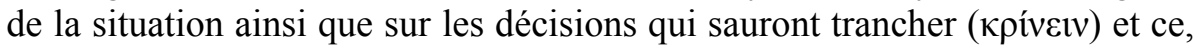
qui plus est, dans le «bon» sens. Le second aspect, l'appel au jugement et aux vertus supposées de l'analyse, est essentiellement lié au premier, c'est-àdire à l'idée que le désordre est une maladie. En effet déclarer l'état de crise revient à juger l'ordre et l'équilibre d'un système en danger, c'est par conséquent d'une capacité de mise en ordre, de clarification, de délimitation et de distinction nette entre le vrai et le faux, le juste et l'injuste que l'on peut attendre une issue heureuse de la crise.

Pourtant ce parti-pris rationaliste ne va aucunement de soi. Sur quels arguments cette lecture prophétique d'une époque troublée se fonde-t-elle ? Il est difficile de dire que, contrairement à ce que Husserl puis Merleau-Ponty - qui reprend en 1958-59 cette théorie de Husserl et jusqu'à la métaphore du phénix — prophétisaient, la philosophie est depuis lors revenue de ces affres pour se montrer dans toute sa gloire. Elle n'est pas davantage morte et enterrée. Le discours de la crise est toujours à l'œuvre aujourd'hui, tant sous ses formes régionales - crise économique, crise de l'éducation, etc. — que sous sa forme globale qui, en fait, sous-tend les premières ${ }^{1}$. La crise dure

(ibid., p. 348 ; tr. fr., p. 382-383). Merleau-Ponty se réfère à ce texte dans les Notes de Cours 1959-1961, Paris, Éditions Gallimard, 1996, p. 39 et p. 72.

${ }^{1} \mathrm{C}$ 'est un des mérites de Husserl d'être parvenu à le démontrer : toute déclaration de crise, aussi circonscrite soit-elle, implique que l'on se demande si le système malade est viable ou pervers par essence. L'appel au jugement inhérent au discours de crise 
singulièrement. Ainsi Paul Ricœur se demande en $1986^{1}$ si nous vivons aujourd'hui "pour la première fois dans l'histoire une crise permanente », question qui résume je crois assez bien le désarroi de notre époque et le caractère usé, peut-être complètement inopérant du concept de crise. Claude Lefort, qui entreprend justement de dissiper ce qu'il définit comme les fantasmes et l'imaginaire de la crise $^{2}$, souligne que le discours de la crise résonne d'autant plus comme le son machinal et inepte d'un disque rayé qu'il n'est pas né d'hier. Lefort en relève ainsi les occurrences répétées au XVIII siècle déjà — dans L'Émile par exemple, «nous approchons de l'état de crise et du siècle des révolutions $»^{3}-$ et tout au long des $\mathrm{XIX}^{\mathrm{e}}$ et $\mathrm{XX}^{\mathrm{e}}$ siècles. Une telle mise en perspective achève de rendre ce discours du moment crucial, du tournant décisif, extrêmement suspect et Lefort se demande ainsi : «Comment un philosophe aussi sobre que Husserl a-t-il pu être à son tour obnubilé par l'idée d'une crise totale et céder au vertige final du ou bien ou bien?» ${ }^{4}$

Toutefois, ce qui me semble le plus digne d'intérêt dans la Krisis de Husserl c'est justement que la déclaration de crise entre en tension avec un deuxième schéma interprétatif beaucoup plus héraclitéen : toute conscience en tant qu'incarnée, est située et aliénée par un écart temporel à soi, par l'étrangeté d'autrui, par des zones aveugles, des influences transcendantes qu'elle ne peut ressaisir et élucider clairement. La réflexion husserlienne dans la Krisis montre également et plus fondamentalement le caractère aventureux et même erratique de tout héritage, la nécessaire infidélité de toute tradition. Ce n'est pas une crise permanente qui nous affecte - l'expression est une contradiction dans les termes - mais plutôt un vertige chronique auquel on ne peut échapper et que Merleau-Ponty va thématiser, subvertissant lui aussi le schéma de la crise qu'il continue pourtant à utiliser. Dans cette seconde approche, on abandonne le discours de crise, discours de celui

va de pair avec la désorientation, le sentiment que nous avons perdu le sens, mais aussi la peur de ne l'avoir jamais vraiment possédé : c'est toujours une crise de la raison.

${ }^{1}$ Dans « La Crise, un phénomène spécifiquement moderne ? » conférence donnée à Neufchâtel, reprise dans la Revue de théologie et de philosophie, vol. 120, 1988, p. 1-19.

${ }^{2}$ Claude Lefort, «L'Imaginaire de la crise », dans Commentaire, vol. 79, Automne 1997, p. 525-536.

${ }^{3} \mathrm{Ou}$, autre exemple célèbre : « Nous touchons à une crise qui aboutira à l'esclavage ou à la liberté », Diderot, Lettre à la princesse Dashkoff du 3 avril 1771.

${ }^{4}$ Claude Lefort, « L'Imaginaire de la crise », art. cit., p. 535. 
qui sait, de celui qui diagnostique et prophétise, pour celui du philosophe boiteux, du cheminement en zig-zag, à travers l'épaisseur des situations.

Ce qui se profile alors prend d'abord la forme d'une ironie permanente: à force de jouer avec les concepts et les points de vue, on sait désormais qu'ils sont tous à double fond, manipulés et trompeurs, et que les manipulateurs eux-mêmes ne peuvent se désengluer de ce champ ambigu de significations anonymes et protéiformes. Personne n'accède à un regard surplombant. Nous contemplons, manipulons et sommes manipulés par des images ${ }^{1}$. Le concept de crise permet certes de surmonter la tentation nihiliste, mais, selon nous, à trop bon compte. Il est, à notre avis, un obstacle à la compréhension de ce qu'il y a de plus audacieux et subtil dans la réflexion phénoménologique sur le malaise actuel. Ainsi cet article s'efforcera-t-il de montrer que les philosophies de Husserl de Merleau-Ponty nous offrent des pistes originales pour instituer, sans espoir de retour sur une terre ferme, une pratique sensée et efficace.

\section{La crise selon Husserl}

C'est la référence à la santé grecque qui fonde le concept husserlien de crise : l'exigence d'auto-méditation (Selbstbesinnung), le " connais-toi toi-même », «l'autonomie théorétique dont découle l'autonomie pratique $»^{2}$, l'autoresponsabilité absolue (Selbstverantwortung) constituent le modèle de santé que Husserl oppose à l'impression contemporaine de perte généralisée de sens. Le projet philosophique grec était en effet celui d'une science omnienglobante au sein de laquelle l'homme découvre comment le monde fait sens, dans tous ses aspects, et peut éclairer chaque décision personnelle. La réflexion doit permettre de découvrir la raison de toute chose et de n'agir qu'en vertu de motifs clairs, ainsi saura-t-elle nous affranchir de toute soumission à un modèle mythique ou à la manipulation sophistique. C'est

${ }^{1}$ Cette menace sceptique est une dimension constamment présente dans les philosophies de Husserl et de Merleau-Ponty, voir par exemple Edmund Husserl, Husserliana VII. Erste Philosophie (1923-1924), Erster Teil: Kritische Ideengeschichte, R. Boehm (Hrsg.), Den Haag, Martinus Nijhoff, 1956 ; trad. fr. A.L. Kelkel, Paris, PUF, 1970, $9^{\text {ème }}$ leçon et Maurice Merleau-Ponty, «Lecture de Montaigne », dans Signes, Paris, Éditions Gallimard, 1960.

2 "Der theoretischen Autonomie folgt die praktische» (Edmund Husserl, Husserliana VI. Die Krisis der europäischen Wissenschaften und die transzendentale Phänomenologie. Eine Einleitung in die phänomenologische Philosophie, p. 6 ; tr. fr., p. 12). 
l'accomplissement le plus ambitieux que la philosophie place à notre portée selon Husserl : «Triompher de l'aveugle moira $»^{1}$. Cette référence offre à Husserl un modèle interprétatif permettant d'identifier les causes de la crise moderne : la maladie est une perte de sens. La tyrannie dont nous subissons le joug est moins celle du mythe que celle d'une science "technicienne » consistant à utiliser des outils d'analyse, de mesure, de calculs, de prédiction et de contrôle relativement efficaces, mais dont l'origine et l'ajustement à la nature profonde des choses nous échappent complètement. Quoi qu'il en soit, la domination l'a encore emporté sur la compréhension et de là proviennent notre exténuation et notre démoralisation. Ce sont donc le positivisme et le naturalisme qui sont à blâmer en tant qu'ils présentent le monde comme un ensemble de réalités en-soi, existant avec leur inertie propre, indépendamment de notre pensée.

Plus précisément encore ce fourvoiement trouve son origine, selon Husserl, dans une reprise de l'idéal grec, débutant à la Renaissance, et qui a perverti l'héritage en croyant lui être fidèle. Retraçons brièvement ce processus, il nous intéresse doublement : par la version critique que Husserl défend explicitement, mais aussi par l'alternative non critique, que sourdement, son récit suggère.

Le monde absolu, idéal est indiqué en horizon de toute perception et $\mathrm{du}$ monde tel qu'il nous apparaît subjectivement. Cet horizon est une dimension à part entière du monde ambiant - sensible - car sans lui nous ne chercherions pas à surmonter certaines perceptions comme étant erronées ou trop subjectives, nous ne partirions pas en quête du monde tel que le voient les autres ${ }^{2}$. Le projet grec de connaissance absolue nous tourne donc de façon privilégiée vers cet horizon. Mais ce monde idéal n'est pas le monde de la vie, il ne se profile qu'en horizon du monde ambiant au sein duquel aucun objet n'est véritablement identique à soi. Aucun corps du monde n'est un cercle parfait ou une ligne parfaite, mais la possibilité de tracer des droites avec une mine de plus en plus fine est bien offerte par ce monde sensible et fait signe en horizon vers l'idée de droite parfaite. La distinction entre idéalité et monde ambiant est encore très claire pour les philosophes grecs, ainsi que le souligne Husserl, et elle est selon lui la clef d'un rapport authentique à l'idéalité. Toutefois, la tentation est grande de passer outre cette manière essentielle qu'a l'en-soi de se donner toujours en horizon, de « faire le saut [hineinspringen] ${ }^{3}$ vers l'idéal et de considérer le

${ }^{1}$ « die die blinde Moira überwunden hat » (ibid., p. 430 ; tr. fr., p. 475).

${ }^{2}$ Ibid., p. 166 ; tr. fr., p. 186.

${ }^{3}$ Ibid., p. 499 ; trad. fr., p. 553. 
monde parfait comme étant une réalité en-soi indépendante du processus spirituel d'idéalisation et de passage à la limite accompli par notre représentation. C'est ce qui se passe lorsque le champ mathématique sédimente en un ensemble d'entités, de figures, de propriétés fixées sur le papier et qui semblent exister objectivement indépendamment de nous. Un nouveau cap est franchi, facilité par cette première hypostase des essences qui a lieu dès l'Antiquité, lorsque l'on substitue ce monde objectif au monde imparfait dont, pourtant, il dépend : ainsi Galilée pense accomplir le projet grec en affirmant que la nature est mathématique. Comme l'origine subjective des idéaux mathématiques est complètement oubliée, la science moderne va avoir les plus grandes difficultés à comprendre pourquoi elle ne peut complètement élucider l'être du monde de la vie. Elle prétend l'étudier, alors qu'elle ne travaille que sur une version schématisée et calculable, version mathématique de ce monde qui lui a été substituée. La dérive se poursuit de façon vicieusement cohérente : en vertu du projet grec de science universelle, le même modèle est appliqué non plus aux corps mais à l'esprit et aux activités et œuvres humaines : l'esprit oublieux essaye, de façon absurde, de se penser comme chose, entraînant alors des difficultés et des paradoxes insurmontables. Les sciences humaines et leur tentative d'étude objective de la subjectivité voient le jour et vivent comme une malédiction inexplicable leur incapacité à atteindre un niveau d'efficacité et de rigueur semblable à celui des sciences de la matière inerte. Husserl souligne enfin le lien essentiel qui unit cette dérive positiviste et une société technicienne présentant certains processus économiques, sociaux, etc., comme fatals et objectifs. Le monde dans lequel nous vivons et qui semble régi par des processus implacables est en fait, dit Husserl, originellement, une vaste construction spirituelle que nous pourrions déconstruire et repenser, mais qui recèle cette possibilité du fait de sa sédimentation en être brut et opaque.

Bref, Husserl veut défendre l'idée que la phénoménologie retrouve l'esprit du projet philosophique originel. En tant qu'elle rattache tout être au sujet transcendantal, elle réalise le projet universaliste grec et sauvegarde l'accès aux essences sans le pervertir. Grâce à la phénoménologie, affirme Husserl, il est possible de retrouver pleinement le sens originel du monde et des concepts. Les proto-fondateurs, les premiers géomètres par exemple, ont, affirme-t-il, éprouvé d'abord "la présence originale et en personne » des idéalités «dans 1'“évidence originaire" " ${ }^{1}$ précisément parce qu'ils avaient

${ }^{1}$ « Das originale Selbstdasein in der Aktualität der ersten Erzeugung, also in der ursprünglichen "Evidenz", ergibt überhaupt keinen verharrenden Erwerb, der objektives Dasein haben könnte », «la présence originale et en personne, dans l'actualité 
conscience du processus d'idéalisation qui présidait à leur formation. Si nous retrouvons la conscience de ce processus animé par le dialogue entre un monde ambiant qui nous parle et une prestation idéalisante qui lui répond, alors nous réactiverons l'intuition absolue et exorciserons la crise.

\section{2. Émergence d'un modèle alternatif à celui de la crise : cercle, zig-zag et vertige}

Les analyses de Husserl esquissent une autre perspective plus angoissante, parce qu'en dévoilant les origines de la dérive positiviste, elles montrent que celle-ci n'est pas un simple accident. Apparaît une certaine logique qui mène d'une étape à l'autre, de sorte que la pureté même de l'origine devient douteuse. Il est en effet impossible de ne pas se demander comment l'évidence de l'intuition originaire, prétendument issue d'une idéalisation en droit parfaitement maîtrisable par toute conscience, a pu se perdre à un tel point. Et d'ailleurs, Husserl concède, au détour de L'Origine de la géométrie, troisième appendice à la Krisis, que les proto-fondateurs ne pouvaient avoir qu'une appréhension incomplète et trouble du contenu et de l'avenir de leurs concepts ${ }^{1}$. Les mésaventures que ces derniers ont connues jusqu'à la crise moderne montrent que l'essence est peut-être nécessairement vouée à demeurer multiple et flottante. Ainsi, la géométrie par exemple, « la mathématique des anciens ne connaît que des tâches finies ${ }^{2}$, " la conception d'une telle Idée d'une totalité d'être rationnelle infinie, systématiquement dominée par une science rationnelle, voilà la nouveauté inouïe [unerhört] » ${ }^{3}$. De même les géométries non euclidiennes prolongent la géométrie euclidienne tout en bouleversant le sens qui était originellement le sien. Ces évolutions inouïes

de la première production, donc dans l'évidence originaire, ne donne lieu, en général à aucun acquis persistant qui puisse avoir l'existence objective » (ibid., p. 370 ; trad. fr., p. 409).

${ }^{1}$ « Der Totalsinn der Geometrie [...] nicht schon am Anfang als Vorhabe [...] dasein konnte », "Le sens total de la géométrie [...] ne pouvait être déjà là dès le commencement comme projet » (ibid., p. 367 ; tr. fr., p. 406).

2 Edmund Husserl, La Crise des sciences européennes et la phénoménologie transcendantale, op. cit., p. 26.

3 «Die Konzeption dieser Idee eines rationalen unendlichen Seinsalls mit einer systematisch es beherrschenden rationalen Wissenschaft ist das unerhört Neue » (Edmund Husserl, Husserliana VI. Die Krisis der europäischen Wissenschaften und die transzendentale Phänomenologie. Eine Einleitung in die phänomenologische Philosophie, op. cit., p. 19 ; trad. fr., p. 26). 
sont bien les fruits de l'institution grecque, de même que, pour prendre un autre exemple d'héritage tout à la fois authentique et faussé, les philosophies de Spinoza et de Leibniz sont les enfants de celle de Descartes ou le second Empire est l' « enfant monstrueux » de la Révolution de 1848.

En conséquence, l'origine doit nécessairement être obscure à ellemême pour produire des rejetons si nouveaux, variés et l'interprétant d'une façon qu'elle ne prévoyait pas ou même, parfois, qu'elle n'approuverait pas et qui pourtant se revendiquent d'elle. L'étude de l'intuition originaire telle que la définit Husserl permet justement d'entrevoir cette idée d'une sédimentation non moins originaire. L'essence se donne en effet originairement comme valable et compréhensible en droit par tout sujet, en tout lieu, à tout moment. En d'autres termes, elle est reconnue comme "présence perdurante $»^{1}$; elle doit être intrinsèquement communicable à autrui et capable d'être fixée durablement comme accessible à tous : c'est donc le passage à une formulation orale puis écrite qui entérine cette objectivité naissante de l'essence ${ }^{2}$. Mais le passage à l'écrit ne saurait être la cause de la sédimentation : nécessairement l'essence sédimente immédiatement, elle est déjà une certaine extériorité relative à l'égard du moi, elle me dépasse, est pour l'avenir et pour autrui. Corrélativement le sujet de l'intuition, en tant que dépassé, se reconnaît comme situé : sa saisie de l'essence est liée à une perspective particulière limitée et à telle ou telle formulation linguistique contingente, tel ou tel conditionnement conceptuel culturel. Le processus de sédimentation décrit par Husserl et déploré par lui n'est donc pas le fourvoiement contingent d'une civilisation manquant son héritage, mais la continuation et le durcissement d'une opacification, d'un écart à soi minant l'origine même. Certes la sédimentation qui semble pouvoir expliquer la crise est par bien des aspects regrettable : elle rend possible incompréhension, mésinterprétation, reprises infidèles, déformations et sentiment d'absurdité, mais elle est également la condition essentielle de l'ouverture du sujet sur une idéalité transcendante et sur les autres ainsi que de l'institution d'un avenir fécond.

Nous pouvons donc voir ici se dessiner un tout autre schème interprétatif de l'histoire et de l'héritage des traditions que celui de la crise. Il s'agit bien d'un motif qui résonne dans le texte de la Krisis, même s'il entre en tension avec la théorie critique qui en est elle aussi un constituant indiscutable. Husserl donne, nous semble-t-il, la plus claire et la plus étonnante

1 « Das verharrende Dasein der idealen Gegenstände » (ibid., p. 371 ; trad. fr., p. 410). ${ }^{2}$ Idem. 
description de ce schéma alternatif dans l'appendice XXVIII, à propos cette fois de l'héritage philosophique ${ }^{1}$. Comprendre une philosophie passée, réactiver son sens, consiste, affirme-t-il, à recevoir sa motivation comme source d'inspiration pour notre propre activité nouvelle, actuelle. Ainsi chacun crée « son poème de l'histoire de la philosophie [seine "Dichtung der Philosophiegeschichte"] $»^{2}$, son «image [Bild] $»^{3}$ de ce qu'est pour lui ${ }^{4}$ la philosophie de Platon, son «Platon non historique», son «Platon non vrai $»^{5}$. Ce n'est pas une création arbitraire, mais puisque la réactivation vivante doit avoir lieu ici et maintenant, il s'agit de ressaisir dans la lecture des œuvres passées un style, une impulsion, qui seront l'axe secret d'une multitude de variations et d'interprétations toutes uniques. D'une façon générale on peut penser - et c'est ce que Merleau-Ponty va entreprendre avec la notion d'institution - tout rapport au passé et à l'héritage sur ce modèle : chaque œuvre d'art, chaque théorie scientifique, chaque loi édictée, chaque acte, chaque décision, chaque perception même est selon une expression merleau-pontyenne "visée à côté qui sera rectifiée " ${ }^{6}$, position d'une question ouverte qui sollicitera à nouveau les divers sujets, suggérant un certain style de réponse, mais suffisamment ambigu pour s'offrir à des « ré-institutions » créatrices éventuellement très surprenantes.

$\mathrm{Au}$ modèle critique, linéaire, peut ainsi être substitué un modèle tourbillonnaire, apparemment vicieux, en tout cas déroutant et vertigineux, d'oscillation sans fin entre des termes qui se conditionnent l'un l'autre et qui se métamorphosent au fur et à mesure qu'on pense les "retrouver» en revenant sur ses pas : la boucle ne peut jamais se clore et le mouvement est

${ }^{1}$ Texte commenté par Merleau-Ponty dans les Notes de Cours 1959-1961, op. cit., p. 85-86 et dans Notes de cours sur L'Origine de la géométrie de Husserl, Paris, PUF, 1998, p. 15 et p. 81 .

${ }^{2}$ Edmund Husserl, Husserliana VI. Die Krisis der europäischen Wissenschaften und die transzendentale Phänomenologie. Eine Einleitung in die phänomenologische Philosophie, op. cit., p. 513 ; trad. fr., p. 568.

${ }^{3}$ Idem.

${ }_{5}^{4}$ Ibid., p. 511 ; tr. fr., p. 567.

5 «Ist seine Arbeit verloren, die er [der philosophische Selbstdenker], um wissenschaftliche Geschichtlichkeit unbekümmert, unter Leitung, unter Verwertung seines "unhistorischen", unwahren Plato etc. getan hat?», «Est-ce que le travail que, sans se soucier d'historicité scientifique, [l'auto-penseur philosophe] a accompli sous la direction de Platon — d'un Platon "non-historique" à lui, d'un Platon nonvrai - est un travail perdu ? » (ibid.., p. 512 ; trad. fr., p. 567).

${ }^{6}$ Maurice Merleau-Ponty, Notes de cours sur L'origine de la géométrie de Husserl, op. cit., p. 30. 
indéfiniment décentré et relancé. Soulignons ici encore que ces figures baroques sont, étonnamment, de Husserl lui-même, toujours dans une réflexion équivoque sur la crise : "Nous sommes donc dans une sorte de cercle. La compréhension des commencements ne peut être obtenue pleinement qu'à partir de la science donnée dans la forme qu'elle a aujourd'hui, et par un regard en arrière sur son développement. Mais sans une compréhension des commencements, ce développement est, en tant que développement-du-sens, muet ${ }^{1}$. «Il ne nous reste qu'une solution, c'est d'aller et venir en "zig-zag" [im "Zickzack" vor- und zurückgehen]» ${ }^{2}$. En effet, puisque le sens d'une théorie ou d'une intuition se dévoile dans les avatars imprévisibles de son héritage, on ne peut espérer comprendre l'origine sans étudier sa postérité. Ainsi Husserl considère que la phénoménologie comprend mieux que Platon ou Descartes eux-mêmes le sens profond de leur projet de science philosophique. Pour résumer de façon un peu abrupte l'idée souvent avancée par Husserl: Descartes voulait une science universelle, il l'a manquée, l'avenir l'a prouvé. Mais, d'autre part, le sens du présent tel que nous le comprenions avant que nous nous en inspirions pour mieux étudier le passé, apparaît enrichi par ce que nous découvrons de son origine, de ses racines. Ainsi Husserl est amené à se méfier d'un processus de fondation de type cartésien, centré sur une réduction, qu'il utilise pourtant à plusieurs reprises. On peut dès lors retourner à l'étude du passé en projetant sur lui la lumière nouvelle d'un présent dont les structures complexes viennent de se dévoiler davantage à nous : en quel sens Descartes était-il sur la bonne voie, pourquoi l'a-t-il manquée, en quoi sa réflexion recèle-t-elle donc une ambiguïté constitutive ? Et en quoi notre réflexion recèle-t-elle donc une ambiguité constitutive ? C'est un mouvement sans fin qui rend par essence inepte le projet de science achevée.

\section{Effondrement de l'analyse critique}

Nous découvrons dans la Krisis une alternative entre deux schémas interprétatifs de la situation de malaise contemporaine. Nous voulons montrer que le

${ }^{1}$ « Wir stehen also in einer Art Zirkel. Das Verständnis der Anfänge ist voll nur zu gewinnen von der gegebenen Wissenschaft in ihrer heutigen Gestalt aus, in der Rückschau auf ihre Entwicklung. Aber ohne ein Verständnis der Anfänge ist diese Entwicklung als Sinnesentwicklung stumm » (ibid., p. 59 ; trad. fr., p. 67-68).

${ }^{2}$ Idem. 
premier, le discours critique, dessert l'intention de Husserl, repose sur un tour de passe-passe rhétorique et, au fond, s'autodétruit.

Ainsi que le note Lefort, le principal défaut du schéma critique est qu'il est possible uniquement si son auteur se place en un point de vue surplombant et prétend désigner le déséquilibre actuel comme une maladie sur fond de référence à un ordre absolu. Le prophète de la crise prétend également savoir que le désordre a atteint un point crucial, que le basculement est imminent et qu'il n'y a que deux alternatives : la guérison ou la mort ${ }^{1}$. En outre, celui qui déclare l'état de crise parle par-dessus la voix des autres, il décrit une situation de confusion dans laquelle des philosophes et des scientifiques se sont trompés sur le sens réel de ce qu'ils pensaient et faisaient, les peuples ont oublié le sens originel du monde, des mots et des concepts qu'ils utilisaient. Le discours de la crise établit artificiellement un hiatus entre la situation de son auteur et la pensée de ceux au nom de qui il parle et qui sont rangés du côté de l'opacité, de la conscience mystifiée. Celui qui maintient le discours de crise crée l'illusion qu'il se tient hors de ce champ ambigu. Il a beau appeler, comme Husserl, à la responsabilité et la réflexion de chacun, il impose à tous un certain cadre conceptuel qui ne va pas de soi.

De là découle également l'usage conservateur et essentiellement nuisible à la démocratie qui a pu être fait de la notion de crise. Si révolution et crise partagent la même référence à des valeurs absolues qu'il s'agit de défendre et si, en outre, une crise peut déboucher sur une révolution, il est frappant de constater que les États et les autorités établies chérissent la rhétorique de la crise. Les dirigeants, conseillers ou autres spécialistes qui l'emploient le font avec une certaine fausse bravoure et une délectation mal dissimulée : cela leur confère en effet, selon un paradoxe de surface, une position de supériorité. Affirmer que nous sommes en crise suggère qu'un ordre existant est menacé, mais peut encore, éventuellement, être sauvé. La société est malade : peut-être découvrira-t-on que, plus radicalement, l'ordre que l'on tente de rétablir n'est pas viable, mais cela n'est pas joué d'avance et il faut aussi essayer de lutter contre la maladie. Face à cette incertitude que la révolution outrepasse - l'état de crise déclaré en appelle au plus

${ }^{1}$ « Mort » dont la teneur exacte demeure assez obscure : il s'agit, peut-être, de l'extinction d'une civilisation, si cela est possible, ou encore de l'installation durable dans une société médiocre, découragée et technicienne. Reste que c'est toujours le modèle de l'ordre, de la clarté et de la juste décision, selon des critères supposés absolus, qui, dans ce modèle critique, sert de référence universelle et de principe de continuité.

61 
grand tact. L'esprit du jugement, le schème directeur de la prudence, de la retenue, de la juste mesure animent essentiellement une déclaration de crise. Il faut s'unir pour lutter contre le désordre qui gangrène nos modèles rationnels; il est judicieux de ne pas multiplier les initiatives débridées et singulières, d'endiguer la violence et de laisser les plus perspicaces coordonner la reprise en main. La notion de crise est ainsi essentiellement conservatrice et anti-démocratique.

En fait ce discours devrait s'autodétruire : la position de surplomb du prophète de la crise devrait être rattrapée et minée par l'indécision quant à la possible valeur du jugement, de tout jugement, mise en évidence par sa théorie. En toute rigueur, le discours critique oblige à rompre avec le discours critique. Celui qui le tient est aussi immergé dans le champ ambigu dont il parle. Le discours de crise ne possède pas de consistance argumentative ; il est une force, une arme, il opère en tant que parole performative qui creuse un abîme fictif entre le prophète, détenteur d'un savoir, et le peuple condamné à errer dans une pensée confuse. Aussi l'autorité politique qui utilise ce discours s'expose tout au plus à former un ensemble de sujets atones, sceptiques et abouliques. C'est pourquoi, nous semble-t-il, la menace du nihilisme est encore à situer du côté du discours de crise. Et si la théorie du cercle ou du vertige permanent peut sembler nihiliste c'est, nous espérons commencer à le montrer, en vertu d'une lecture superficielle.

Il nous faut en effet maintenant examiner la principale objection faite au modèle du vertige permanent: son caractère ruineux pour la pensée et l'action. Ne doit-il pas immanquablement nous plonger dans la stupeur et le découragement? Nous voulons montrer que tout l'intérêt de la phénoménologie est de nous donner au contraire les moyens de penser ce vertige sans sombrer dans le nihilisme.

\section{Agir quand même, sans le secours du modèle critique : pour une phénoménologie de l'action symbolique}

Certes le temps linéaire et ordonné du modèle critique a laissé la place à un temps tourbillonnaire, concédons néanmoins que cela rend impossible l'irruption d'un moment de décrochage, de nouveauté absolue, de ce trésor de la liberté dont parle Hannah Arendt dans La Crise de la culture, le décrivant comme un héritage sans testament et dont la crise, comme effondrement des schémas habituels, favoriserait l'épiphanie : celui qui se croit visité par la liberté même, par la certitude — échappant à toute 
formulation ou réflexion, dit Arendt - certitude d'avoir découvert sa vérité ${ }^{1}$, d'«aller nu», «dépouillé de tout masque »", est encore conscience mystifiée. Ce discours arendtien dont la tonalité est encore critique - et à la complexité duquel nous ne pouvons rendre justice dans le cadre restreint de la présente étude - est donc en conflit avec le modèle du vertige permanent. Dans le flux héraclitéen, j'ai certes une marge d'interprétation, de décision certaine ici et maintenant, mais avec les moyens du bord, dans une situation, avec des outils conceptuels, linguistiques et techniques que je n'ai pas fondés, et ce que je produis sera immédiatement récupéré et repris, prolongé et déformé par le monde et par les autres. La temporalité du vertige permanent est celle d'une ubiquité en vertu de laquelle je m'aperçois diffracté entre le passé et l'avenir, les passés et les avenirs, les images incertaines de ce que j'étais et de ce que je serai, chacune se reflétant de façon déformante dans les autres. Se profile alors très sérieusement le risque d'une relative indifférence du moment présent. Le cercle dont parle Husserl, le thème de l'éternel retour chez Merleau-Ponty ${ }^{3}$ ne nous condamnent-ils pas au dégoût de l'action?

Nous ne le croyons pas. La temporalité vertigineuse qui apparaît dans la Krisis est aussi fondamentalement une philosophie de l'héritage. Husserl ne démontre nullement mon aliénation, passivement subie, par un passé et un futur que je ne suis pas, mais élabore au contraire une nouvelle conception d'une diffusion de chaque moment à travers les autres sous la forme d'une Stiftung, d'un appel à comprendre en créant. Parce qu'il n'y a pas de contenu d'intuition originaire parfaitement circonscrit, mais d'emblée des significations se profilant en horizon d'une pensée temporelle en quête d'elle-même, l'héritage ne peut être saisi fidèlement à la façon dont on met la main sur une chose. Inachevé et prospectif dès l'origine, le sens est une «impulsion

\footnotetext{
${ }^{1}$ Hannah Arendt, La Crise de la culture, trad. fr. P. Lévy, Paris, Éditions Gallimard, 1972, p. 12.

${ }^{2}$ Idem.

${ }^{3}$ « Ce qu'il y a c'est non série, mais emboîtement : le présent (toujours sensible et toujours spatial) tient dans sa profondeur d'autres présents [...]. La généralité du temps, le concept de temps (qui n'est pas seulement irréversibilité mais aussi éternel retour: il n'est autre que parce qu'il est le même) qui est vécue jusque dans le présent, tiennent à cet emboîtement à l'intérieur du présent » (Maurice MerleauPonty, Notes de cours 1959-1961, op. cit., p. 208-209. Voir aussi ibid., p. 375 : « [La philosophie] c'est l'invitation à re-voir le visible, à re-parler la parole, à re-penser le penser », ainsi que Maurice Merlau-Ponty, Le Visible et l'invisible, Paris, Éditions Gallimard, 1964, p. 233.
} 
[Impulse] ${ }^{1}$ qui relance l'interprétation, laquelle ne tourne pas vainement autour d'une vérité en-soi dont l'accès lui serait interdit, mais s'ouvre au sens passé en acceptant la responsabilité de le faire vivre dans un corps nouveau ici et maintenant: la compréhension poétique est créatrice sans être arbitraire $^{2}$.

La fin de la crise ne peut ainsi advenir que de notre capacité à penser ensemble deux positions que le discours de crise sépare indument : d'un côté la situation du prétendu prophète de crise, de celui qui analyse, accède au sens et, d'un autre côté, celle du peuple mystifié et désorienté. En d'autres

${ }^{1}$ Edmund Husserl, Husserliana VI. Die Krisis der europäischen Wissenschaften und die transzendentale Phänomenologie. Eine Einleitung in die phänomenologische Philosophie, op. cit., p. 511 ; tr. fr., p. 567.

${ }^{2}$ «Der philosophische Selbstdenker [...] nimmt was sich ihm als Tatsache der Überlieferung darbietet, unkritisch als solche hin und lässt sich von dem, was er theoretisch hineinversteht, von "der" platonischen, Aristotelischen Philosophie motivieren. », «L'auto-penseur philosophe [...] reçoit sans critique ce qui s'offre à lui comme un fait de tradition et il se laisse motiver par ce qu'il y comprend théorétiquement et qui est pour lui "la" philosophie platonicienne, aristotélicienne etc. » (ibid., p. 511 ; trad. fr., p. 566-567). « Und philosophierend ist er auch mit sich selbst, alswie er Philosophie früher verstand und betrieb, in Konnex, und er weiß, daß dabei immer historische Tradition, so wie er sie verstand und benützte, im ihn motivierend und als geistiger Niederschlag einging. Sein Historisches Bild, teils selbstgebildet, teils übergenommen, seine "Dichtung der Philosophiegeschichte" blieb und bleibt nicht fest, das weiß er; und doch: jede "Dichtung" dient ihm und kann ihm dienen, sich selbst und sein Absehen, und das seine in bezug auf das der Anderen und ihre "Dichtung", ihr Absehen, endlich das Allgemeinsame zu verstehen, das "die" Philosophie als einheitliches Telos, und die Systemversuche seiner Erfüllung für uns alle, für uns zugleich in Konnex mit den Philosophen der Vergangenheit (so wie wir sie uns vieldeutig dichten konnten) ausmacht», «Et philosophant ainsi il est également en connexion avec soi-même, avec sa façon antérieure de comprendre la philosophie et d'en faire, et il sait que dans ce processus la tradition historique, telle qu'il l'a comprise et utilisée, n'a jamais cessé d'intervenir et de le motiver spirituellement. L'image qu'il se fait de l'histoire, en partie forgée par lui-même, en partie reçue, son "poème de l'histoire de la philosophie" n'est pas resté et ne reste pas fixe, il le sait; et pourtant: chaque "poème" lui sert, et peut lui servir, à se comprendre lui-même et son projet, et celuici en rapport avec celui des autres et avec leur "poème", et finalement à comprendre le projet commun à tous, qui constitue "la" philosophie en tant que télos unitaire avec les tentatives systématiques de remplissement de sens pour nous tous, c'est-àdire en même temps en connexion avec les philosophes du passé (pour autant que nous puissions en donner diverses "versions poétiques" qui aient du sens pour “nous") » (ibid., p. 513 ; trad. fr., p. 568). 
termes, il s'agit de penser comme rapport authentique au sens et fécondité pratique, l'interprétation, l'angoisse, l'ambiguïté et le jeu aventureux avec les images. À la question ouverte par Husserl - comment penser la connaissance et l'action d'un même mouvement et sur le modèle de la poésie ? Merleau-Ponty élabore une réponse qui n'est plus thématisée comme marge de la phénoménologie, mais bien comme sa thèse essentielle. L'un de ses concepts clefs est celui d'action symbolique.

« Il n'y a que des actions symboliques $»^{1}$ affirme Merleau-Ponty dans Les Aventures de la dialectique. Voilà qui ne saurait manquer de nous faire douter à nouveau : serions-nous donc replongés dans le nihilisme ? La notion merleau-pontienne d'action symbolique est à première vue décevante en ceci qu'une connotation péjorative est habituellement attachée à cette expression : l'action symbolique est communément un acte vain, incapable de produire en tout cas comme son résultat visible immédiat - une transformation effective de la réalité. Merleau-Ponty assume cet aspect de l'action symbolique : emportés par un sens toujours en décalage avec lui-même, héritiers de questions plus que de certitudes, dépendants des interprétations que les autres produiront de nos paroles et de nos actes, nous ne pouvons en effet jamais espérer produire un résultat parfaitement maîtrisé ${ }^{2}$.

Merleau-Ponty accomplit en fait ici une sorte de « dégonflement» des concepts traditionnels positivistes : il montre qu'une telle dimension symbolique peut, malgré et même grâce à son manque d'éclat et d'efficience brute, devenir féconde en vertu d'un déplacement de l'action au niveau de la quête de sens en commun.

Comme nous l'avons vu, il s'agit pour Husserl, comme pour MerleauPonty et dans l'esprit du mouvement phénoménologique, de conserver notre lien au sens. Certes, ce dernier est désormais compris comme ambigu, sujet à interprétation, mais également comme constitué par des lignes de saillance, des axes directeurs s'esquissant au creux de la diversité des phénomènes, de sorte qu'il est possible d'être plus ou moins habile dans l'art de les lire et de s'y adapter et de les exploiter.

C'est exactement ce que la théorie husserlienne de la sédimentation et de l'héritage démontre: un sens résonne sourdement dans des lignes de transmission aventureuses au sein desquelles on peut lire une certaine cohé-

\footnotetext{
${ }^{1}$ Maurice Merleau-Ponty, Les Aventures de la dialectique, Paris, Éditions Gallimard, 1955, p. 250. Voir également Edmund Husserl, La Crise des sciences européennes et la phénoménologie transcendantale, op. cit., p. 279.

2 « Nulle action n'assume tout ce qui se passe, n'atteint l'événement même » (ibid., p. 278-279).
} 
rence, une direction commune, sans que, pour autant, une loi positive puisse être découverte qui rendrait l'enchaînement des événements nécessaire. De Socrate à Galilée, en passant par les sceptiques et Descartes, il y a bien une certaine histoire, un certain héritage idéaliste qui se continue, mais un héritage problématique, chahuté et ouvert à des interprétations diverses de sorte que des surprises adviennent sans cesse et que l'avenir lui-même reste indéterminé.

Dans Les Aventures de la dialectique, Merleau-Ponty montre que cette structuration de l'histoire par des lignes ambiguës n'est pas un phénomène de surface résultant de la finitude de notre perspective humaine. C'est la nature absolue de toute existence et de tout monde possible: le monde, comme diversité d'êtres et comme une unité de sens, ne saurait apparaître qu'à des subjectivités incarnées. Le monde doit être cette diversité de points de vue et de perspectives, certes diffusant les uns dans les autres, mais qui ne coïncident pas nécessairement. Un Esprit omniscient s'auto-contemplant dans une complétude et une béatitude absolues ne serait pas un monde. Dès lors Merleau-Ponty peut avancer la thèse métaphysique selon laquelle il n'y a pas une "Raison de l'histoire» de type hégélien, mais bien plutôt une « imagination de l'histoire $»^{1}:$ le sens des événements et des institutions est en lui-même indécis. Déjà dans la Phénoménologie de la perception, Merleau-Ponty montrait que le prolétariat n'est jamais un être positif, qu'il est une entité spectrale éparse se formant via un phénomène d'écho incertain, entre une multitude de micro-événements singuliers ${ }^{2}:$ «Le journalier a perçu concrètement le synchronisme de sa vie et de la vie des ouvriers [...]. Le petit fermier [...] se sent solidaire des ouvriers quand il apprend que le propriétaire de la ferme préside le conseil d'administration de plusieurs entreprises $»^{3}$. Les situations individuelles se répondent et un sens s'annonce entre les actes de chacun, "en filigrane ${ }^{4}$ : un tel sens dépasse chaque individu particulier et constitue une force capable de porter les projets de chacun ou de les faire échouer. Mais il est aussi constitué par l'approche de chacun. Par conséquent, une marge de manœuvre individuelle subsiste. En tant que sens épars dans une multitude de variations concrètes, de points de

${ }^{1}$ Maurice Merleau-Ponty, Les Aventures de la dialectique, op. cit., p. 29, p. 53 et p. 183.

${ }^{2}$ Il s'agit, dit Merleau-Ponty, d'un « processus moléculaire » (Phénoménologie de la perception, Paris, Éditions Gallimard, 1945, p. 509). Il serait intéressant de montrer qu'à bien des égards une telle théorie pluraliste et poétique du sens annonce l'approche deleuzienne.

${ }^{3}$ Ibid., p. 507-508.

${ }^{4}$ Maurice Merleau-Ponty, Les Aventures de la dialectique, op. cit., p. 28.

66 
vue, de subjectivités, en tant que fantôme qui hante l'Europe, le prolétariat est un sens encore en chemin et qui se module aussi en fonction des diverses individualités qui l'esquissent et tentent de le penser et de le réaliser, chacune à sa façon.

Tel est donc le contexte, inconfortable mais confusément sensé, de l'action. Dans ce cadre, que peut-on connaître et comment agir ? Les deux questions s'entrecroisent: il n'y a pas d'objets achevés à connaître; la manière dont nous allons interpréter le monde et les événements, ainsi que nos actions, vont continuer à modeler la réalité. Ce que Merleau-Ponty met en place est par conséquent pleinement une philosophie de l'action, en un sens renouvelé : une philosophie dans laquelle gnôsis, praxis et poïsis ne peuvent plus être séparés ${ }^{1}$.

Tout d'abord, puisque l'histoire est secrètement structurée par une imagination de l'histoire, puisque des lignes de sens s'esquissent, on a bien les moyens conceptuels de fonder une théorie de l'action éclairée, en tout cas habile, la plus habile possible. Il est possible d'apprendre à lire les circonstances, l'enchaînement des événements, le sens enfoui dans la langue, les institutions, les infrastructures, les symptômes médiatiques, etc. Nous pouvons ainsi - et il le faut autant que possible - agir en nous interrogeant sur la capacité du contexte à porter notre action, à la reprendre ou à la renverser en son contraire. « De ce que je vis, j'aurai demain à construire une image et je ne peux, au moment où je le vis, feindre de l'ignorer $»^{2}$. «Le savoir et l'action sont deux pôles d'une existence unique ${ }^{3}$. Agir c'est toujours « connaître », il n'y a pas d'efficacité envisageable sans l'art de lire les significations et tendances qui sont à l'œuvre actuellement - l'imaginaire de la situation - et sans une imagination de la manière dont l'acte que nous projetons va réagir au contact d'un tel contexte.

Mais ce n'est là qu'un versant de l'action et on ne saurait la réduire à un tel calcul sous peine de défendre toutes les formes d'opportunisme. En

${ }^{1}$ Il ne s'agit ainsi nullement d'une philosophie de l'action par rupture avec une philosophie de la connaissance (schéma qui était suggéré par Arendt à propos de l'existentialisme), ni d'une position prudente de retrait contemplatif que semblait décrire la théorie de la "présence absente » du philosophe que Sartre avait vivement critiqué lors de la dispute de 1953 : «Ta présence au Comité de Défense des Libertés est beaucoup trop songeuse pour qu'on la pense efficace » (Jean-Paul Sartre, Lettre à Maurice Merleau-Ponty du 18 Juillet 1953, Magazine littéraire $\mathrm{n}^{\circ} 320$, avril 1994, p. 71). Merleau-Ponty élabore dans Les Aventures de la dialectique une philosophie de l'action poétique.

${ }^{2}$ Maurice Merleau-Ponty, Les Aventures de la dialectique, op. cit., p. 19.

${ }^{3}$ Ibid., p. 20. 
fait, si agir c'est connaître (au sens d'interpréter), alors, réciproquement, interpréter c'est toujours déjà s'engager et agir. En 1940, il ne s'agit pas seulement de savoir si la défaite de la France contre l'Allemagne est sans issue. Il s'agit de lire quelles lignes de probabilité se dessinent et cela encore n'est pas déterminant : les résistants ont parié sur une victoire de la France pourtant très peu esquissée et même démentie par les faits ${ }^{1}$, et leur pari est un engagement qui a permis à cet axe d'interprétation de se réaliser. Il a fallu agir pour défendre ce pari. Rien ne garantissait le succès de l'entreprise par ailleurs. Imaginer l'avenir d'une action ne consiste donc pas simplement à s'adapter à ce que la situation semble permettre, c'est élaborer en poète, en artiste, la capacité de son œuvre à résonner sur le long terme et dans le plus d'existences diverses possibles. C'est ce point précis qui fonde la notion d'action symbolique.

Toute action «escompte, autant que des résultats immédiats dans l'événement, l'effet qu'elle fera comme geste significatif et trace d'une intention $»^{2}$. Puisque la réalité où s'inscrit l'action et qu'elle veut modeler est fondamentalement de l'ordre de la signification, alors l'action la plus opérante est aussi la plus profonde significativement; elle vise le sens plutôt que la réalité prosaïque, déjà sédimentée : un livre peut ainsi être plus fécond qu'une émeute. « La règle de l'action [...] n'est pas d'être efficace à tout prix mais d'abord d'être féconde $»^{3}$ : l'efficacité est pensée en termes de résonance à long terme dans le champ de la signification. Merleau-Ponty invoque en conséquence une politique qui assumerait son œuvre d'interprétation et s'offrirait au jugement de l'histoire, c'est-à-dire de tout sujet présent et à venir. Il s'agit donc de s'adresser à un sujet irréel, aux jugements les plus divers émis selon des points de vue multiples, bien au-delà des normes et des perspectives actuelles. "L'histoire est juge, - mais non pas l'histoire comme Pouvoir d'un moment ou d'un siècle ${ }^{4}$. Ce juge n'est pas exactement l'incarnation de ce que nous croyons être la loi morale, la justice bien comprise : Merleau-Ponty y insiste en effet, il n'y a pas de point de vue universel, pas d'absolu possible, le monde et la pensée ont «plusieurs centres $\rangle^{5}$ et la synthèse est impossible. L'universel, au-delà d'ailleurs même du concept d'humain, ne se définit jamais par un contenu, mais par une quête

${ }^{1}$ Maurice Merleau-Ponty, Humanisme et terreur. Essai sur le problème communiste, Paris, Éditions Gallimard, 1947, p. 42.

${ }^{2}$ Maurice Merleau-Ponty, Les Aventures de la dialectique, op. cit., p. 279.

${ }^{3}$ Maurice Merleau-Ponty, Signes, op. cit.. p. 91.

${ }^{4}$ Ibid., p. 92.

${ }^{5}$ Maurice Merleau-Ponty, Les Aventures de la dialectique, op. cit., p. 283. 
commune, un dynamisme d'échange. Cet « universel latéral ${ }^{1}$ vit dans le dialogue incessant entre points de vue et modèles culturels, politiques et éthiques différents. Notre conscience est partielle, toujours mystifiée Merleau-Ponty le concède à l'approche marxiste - mais cela signifie indissociablement qu'elle pressent ce qui la limite, elle n'accède pas à la vérité - un tel absolu n'existe pas - mais a le pouvoir de se décentrer indéfiniment et de s'ouvrir à l'altérité, au monde et aux autres d'abord en apprenant à voir comme étranger ce qui lui était le plus familier ${ }^{2}$. Ainsi faire de l'histoire le juge de notre action ne consiste pas à nous adresser au sujet moral idéal, mais bien plutôt à raviver la pensée sauvage ${ }^{3}$, que MerleauPonty appelle également « la fonction symbolique ${ }^{4}$, c'est-à-dire le pouvoir de se transposer d'une perspective à l'autre. La communauté n'a de fragile consistance que dans le procès d'une telle dynamique. L'action symbolique attire ceux à qui elle s'adresse sur un « terrain » d'aventure commun, où nous pouvons tous nous retrouver en ce que nous cherchons l'absolu. Elle se reconnaît donc à ceci qu'elle laisse apparaître la part d'interprétation et d'hypothèse qu'engage toute action. Ainsi Merleau-Ponty invoque la figure de Max Weber dont le «libéralisme est militant, souffrant même, héroïque, c'est-à-dire qu'il reconnaît le droit de ses adversaires, ne consent pas à les haïr, n'élude pas la confrontation avec eux et ne compte, pour les réduire, que sur leurs contradictions propres et sur la discussion qui les révèle $»^{5}$.

\footnotetext{
${ }^{1}$ Maurice Merleau-Ponty, Signes, op. cit., p. 150.

${ }^{2}$ Merleau-Ponty donne en modèle l'ethnologue dans « De Mauss à Lévi-Strauss » : en nous transposant dans d'autres cultures ainsi que le fait l'ethnologue, nous apprenons également à « voir comme étranger ce qui est nôtre » (ibid., p. 151).

${ }^{3}$ Ibid., p. 151.

${ }^{4}$ Ibid., p. 153.

${ }^{5}$ Maurice Merleau-Ponty, Les Aventures de la dialectique, op. cit., p. 41. MerleauPonty donne peu d'exemples d'actions symboliques. Comment organiser exactement la vie politique suivant ce modèle ? Quel type d'État peut parvenir à relancer incessamment un dialogue ouvert sans voir ses institutions ruinées par une telle instabilité et par la récusation de toute valeur absolue et même le refus de supposer quelque réalité positive que ce soit ? On peut trouver, dans «Demain... », avec la référence à Pierre Mendès-France, quelques indices supplémentaires permettant d'esquisser le modèle politique envisagé par Merleau-Ponty (Signes, op. cit., p. 434), mais la référence reste extrêmement succincte et il faut convenir que Merleau-Ponty nous lègue une pensée politique encore très largement «en chantier». Nous ne pouvons développer ici davantage la réflexion en ce sens, mais nous aimerions signaler une piste qui nous semblerait de tout premier intérêt : il y a, croyons-nous, plusieurs traits de parenté remarquables entre le projet merleau-pontien ébauché autour de la notion d'action symbolique et le projet de constitution, cette fois tout à
} 
Ainsi le vertige radical de l'existence n'est nullement atténué, il est intégré à une action audacieuse, difficile, qui entraîne autrui dans une communauté dynamique ne tenant que par le dialogue - parce que toute assise positive, dans quelque réalité ou valeur absolue que ce soit, a été dissoute.

fait articulé et concret, exposé par Bruno Latour dans Politiques de la nature (Paris, Éditions La Découverte, 1999). Partant de la remise en question radicale de la référence à une réalité achevée, nouant indissociablement politique, science et morale, Latour appelle la formation d'une démocratie dans laquelle la parole serait accordée aux non-humains comme aux humains, via des procédés de "phonation », de collecte de la parole et de re-présentation élaborés au cas par cas dans les pratiques politiques mais aussi scientifiques et selon un souci de respect de tous les êtres interrogés. Notre parole est toujours une extériorisation passant par des choses et, réciproquement, aucune chose n'est muette puisque les êtres qui composent notre monde n'existent pas en soi, pas plus d'ailleurs qu'ils ne sont forgés arbitrairement. Les êtres sont des «propositions » (Merleau-Ponty dirait un sens esquissé, une ligne mélodique encore indécise) qui appellent un travail créatif d'élaboration grâce à la formation de concepts, d'œuvres d'art, de techniques diverses, d'instruments de représentation, d'expérimentation et de modélisation scientifique, à travers lesquels les choses mêmes parlent ou, plus exactement, dialoguent avec nous, tout en évoluant et cristallisant sous des formes inédites en vertu de ce dialogue même. Il s'agit alors, selon Latour, d'inventer une démocratie dans laquelle seraient mises en place les conditions concrètes d'une discussion en commun, sans exclure a priori quelque possible intervenant que ce soit, discussion dans laquelle les choses et les animaux s'avanceraient également entourés de leurs amis, parrains, représentants, experts et garants (p. 231) et pourraient prendre la parole. Le processus de création en commun d'un cosmos à travers des discussions dont l'issue ne doit pas être jouée d'avance ni même encadrée par des valeurs prédéterminées devient ainsi le cœur dynamique, le cœur d'instabilité assumée, absolument publique, d'une démocratie authentique et c'est, selon Latour, dans le développement d'une capacité généralisée à se décentrer (jusqu'à se décentrer dans les choses, à un niveau d'ouverture politique que Merleau-Ponty n'envisageait pas mais que sa philosophie de chair du monde justifierait parfaitement), à « décaler sans arrêt le point de vue » (p. 191), que le « collectif », comme collecte de parole, pourra prendre corps. C'est une véritable action symbolique au sens merleau-pontien qui trouve, nous semble-t-il, sa possibilité concrète dans un tel projet : l'élaboration du sens et celle de la réalité et $\mathrm{du}$ monde commun vont de pair et sont donc toujours des affaires politiques, affaires de discussion et de négociation pures. Nous renvoyons à ce passionnant et audacieux ouvrage pour la description détaillée de la nouvelle Constitution envisagée par Latour. 


\section{Conclusion}

Le concept de crise pouvait sembler le plus indiqué pour en appeler à un sursaut rationnel salutaire à un moment où le relativisme et le nihilisme gagnent du terrain. C'est, me semble-t-il, dans cet esprit que Husserl et Merleau-Ponty l'utilisent. Aussi sommes-nous surpris de voir de quelle façon, aujourd'hui, le discours de la crise est chronique, paralysant et conservateur. On pourrait prétendre que la faute en revient à une mauvaise compréhension de ce concept de crise. Nous avons essayé de démontrer que tel n'est pas le cas et que ce concept même doit être abandonné. Nous n'allons ni guérir ni mourir de la crise qui, du coup, n'est pas vraiment une crise. Le doute, la découverte de l'instabilité des valeurs ne sont pas des maladies mais des vertus. L'héritage est certes une source d'inspiration, mais il est d'abord et essentiellement une invitation à la créativité et à l'audace sans aucune garantie ni modèle absolu.

Il n'y aura donc pas de fin de la crise selon le sens défini par le concept de crise. Le concept même de fin - comme s'il pouvait s'agir de trancher - est encore un peu trop « critique ». L'une des conclusions qui découlent de nos analyses est donc aussi que le discours de crise a encore de beaux jours devant lui : l'ambiguité et le vertige existentiels en feront toujours une issue illusoire tentante. Reste que nous pouvons le dénoncer comme truqué, comme l'arme déguisée de tendances antidémocratiques, arme d'autant plus efficace qu'elle est déguisée en voix de la raison. Nous pouvons alors envisager une autre « fin » de la crise, un affranchissement de ce modèle, qui sait par ailleurs qu'il ne pourra jamais se tenir pour acquis. 